\title{
A Freelancer's Approach to Teaching Cartography
}

Daniel P. Huffman daniel.p.huffman@amail.com

While I mostly make my Living as a freelance cartographer, over the past several years I've also had the privilege to serve intermittently as a lecturer for an introductory cartography course at the University of Wisconsin-Madison. Through my simultaneous development as both an educator and a practitioner, I've slowly changed my classroom approach to better reflect my experiences in the real world of mapmaking. Although I cover the typical roster of topics in my course, it is structured in a way that is informed by my own hands-on perspective. This paper includes an à la carte selection of some elements that reflect this practical approach, and which I have found successful in helping students learn. Perhaps the ideas I explain here will inspire a more enterprising instructor to put them to a formal test.

First, a quick bit of background on the course, which is titled "Introduction to Cartography," and enrolls anywhere from 30 to 80 students depending on the semester. I give two 75-minute lectures per week, which cover broad mapmaking fundamentals, including typography, projections, colors, visual variables, etc., as well as the critical thinking skills needed to make good cartographic choices. Students also meet once a week in smaller (15 to 20 person) lab sections, which are conducted by a graduate teaching assistant who guides students in learning hands-on technical skills in ArcMap and Adobe Illustrator. Students also complete mapmaking assignments during and outside of lab. No prior knowledge of GIS or mapping is required to take the course. My objective is to see students come out of the course with the capacity to make basic maps on their own, and to critically assess maps they encounter in their daily lives.

\section{EMBRACING SUBJECTIVITY}

In my freelance work, there is no single, absolute, right way to make a map. Any two people may disagree on what works and what looks good, and whatever pleases one of my clients (or colleagues) may displease another. Likewise, grades on mapping exercises are, in essence, opinions. The person assigning the grade gives their opinion on how well the student has addressed a problem that has a virtually infinite range of possible solutions, and that opinion may vary among graders. I long struggled with the subjectivity inherent in assessing students' work before I decided to simply embrace this fact and own it in front of my students.

It's important to me to begin the course by telling students that their grades are based on my opinion and on the opinions of the teaching assistant(s). I tell them to think of us as their clients: the work they do has to make us happy and conform to what we think is good practice. I acknowledge that they may disagree with us at times, but that they need to set aside their view and make the client's perspective the priority, just as I do in my freelance practice. At the same time, I empathize with students, telling them how my work often requires me to shelve some of my preferred ideas in favor of the wishes of others.

I do not pretend to them that cartography is objective, or that it has absolutely correct answers; instead I explicitly state the opposite at the beginning of the course and in the syllabus. I want students to understand that it is not automatically wrong to go against the ideas I present in lecture. At the same time, I acknowledge the reality that it is my course, and we need to do things my way (even if it is not the only valid way). In this way, I sidestep fruitless arguments about our individual subjective preferences.

Subjectivity is also reflected in the way grades are assigned. Rather than using a system of points, all assignments simply receive letter grades. To me, it seems rather clearer to tell a student that they got a B than to tell them that they got 10 out of 14 points on an assignment: this helps them realize better where they stand. With a simple number grade, it can be difficult at first glance for 
a student to tell if they've done a good job, or failed to meet expectations. Given how subjective grading can be, points also carry an inappropriate air of precision. At the end of the semester, the university requires that I summarize a student's entire course performance in a single letter grade, which represents my opinion on how well the student has learned mapmaking. If students have been seeing letter grades on their assignments all along the way in the course, then the end result is less surprising to them. Since instituting this system, I have indeed seen fewer students push back against their final course grades.

I do have to use point values internally for practical reasons, so that I can average or add together assignment grades. I convert letter grades to numerical grades using the university's four-point scale $(\mathrm{A}=4, \mathrm{AB}=3.5, \mathrm{~B}=3$, $\mathrm{BC}=2.5$, etc.). To make the process easier for students, I provide them with an online grade calculator. They can enter the letter grades from each of their assignments, and it calculates their current course grade in letter format.

\section{NO MORE EXAMS}

Probably the boldest move I've made as an educator is to eliminate quizzes and exams from my course. My freelance clients evaluate me solely on what I produce, and I have come to believe that my students should likewise be graded on the quality of the maps they make, rather than how well they can parrot the content of my lectures. My goal is to give students the skills to independently practice cartography. Either what I teach them will help them make better maps during their lab assignments, or they shouldn't be asked to remember it.

My lectures have value: I teach fundamental concepts, discuss good practices, and lead students in group critiques of published professional maps. They then get a chance to demonstrate, via their graded lab exercises, that they've absorbed that material-or at least picked it up from somewhere else. I primarily want to see what the students can make; I am less concerned with how they learned it, whether from listening to me, or browsing cartography blogs, or intuiting good practice on their own. However it works out, I believe their grade in the course should primarily reflect their ability to create maps. Over half of students' grades come from their five lab assignments, and another third comes from a final project map that they complete at the end of the semester.

\section{SECOND CHANCES}

In my freelance work, I make many drafts. A client will ask me to create something, I will show them an initial attempt, and then I will make revisions based on their feedback. Often I'll even do a revision or two before clients see the work, in response to critique from colleagues. The first version I make is rarely the final, and there is generally no penalty for not getting it right on the first try.

I think students deserve the same chance to revise their work. Having their final grade depend on a first effort is not reflective of how cartography (and many other pursuits) works in the real world. Therefore, after a student has received their graded map, they can revise and resubmit it within two weeks for a new grade. About a third of all lab assignments are revised under this system; for the rest, the students are sufficiently content with their grade that they don't make a second attempt. In my first semester, I limited students to a single revision per lab assignment. The second time around, I gave them a total budget of five revisions for the semester (so that they could revise a lab multiple times while leaving others untouched); however, I have only seen one student revise a map a second time.

There is an incentive to do better on the first try: if a student's initial this grade was a $\mathrm{C}$ or worse, then their final grade for the lab will be the average of the revised grade and the initial grade. If their initial grade was better than a $\mathrm{C}$, then their final grade will simply be the grade of the revised map, and the old one will be discarded. This minimizes a possible abuse of the system, in which a student might use a revision to give themselves a deadline extension on an assignment by first turning in an incomplete map, then finishing it and turning it in as their revised copy later on.

This system of lab revisions not only allows but encourages students to learn from their mistakes, and it has been successful in doing so. In my two most recent semesters of teaching Introduction to Cartography, the average lab received 3.4 out of 4 points (so, about an $\mathrm{AB}$ in our letter-to-number conversion). In the two semesters prior to that, in which lab revisions were not allowed, the average lab grade was 3.0 points (a B), 13\% lower. To be fair, these numbers don't come from any controlled study: these courses took place at different times, with different teaching assistants grading the labs, and so on. But, I think the results are quite encouraging, and suggest that students 
are indeed coming out of the class with a better ability to make maps.

I originally added lab revisions to compensate for the fact that I eliminated exams. Since students' course grades now depend primarily on their lab assignments, they have a lot of eggs in one basket. There are only five labs, and a misstep on any one could do real damage to someone's final grade. Giving students a second chance minimizes that impact, letting them correct a stumble here or there. I do not wish to overly penalize students as they make mistakes on their way to developing skills; I only care that they develop them.

\section{BEHIND THE SCENES}

A number of my students each semester have an interest in continuing on professionally in mapmaking; yet it can be difficult for them to get a sense of what cartographers actually do in the structured and controlled environment of a college course. So, I try to offer glimpses into my freelance career by taking a few minutes during lecture to show them projects I'm working on. In the past I have shown them how I interact with clients, and how, in my own work, I implement the the good practices I demonstrate in lecture. In addition, I show them how my work must sometimes change in response to client requests (again, trying to empathize with them regarding the need to please others), as well as feedback from colleagues, showing how the process of improvement doesn't stop once you become a professional.

When I take these side detours into my freelance work, I get more questions and engagement from the students than almost any other time in the course. They want to know more about what it's like to have a cartography job: How much do I make? How do people find me? How do I figure out how much to charge for a project? How long does it take me to make a map? Do cartographers have meetings? What other kinds of jobs are there in cartography? These are important questions, and they're ones that don't always fit into my normal lecture content, which focuses more on the details of how to make a proportional symbol map or on how to make good typographic choices. I have seen how eager the students are to know how mapmaking works out there in the real world.

During the final lecture period of the semester, instead of talking at the students, I do a live demo. I make a simple map, from start to finish, over the course of about 70 minutes, so they can see my own personal mapmaking workflow. When I go to conferences or read articles in Cartographic Perspectives, I learn a lot from watching my colleagues show their processes and share their tips and tricks. Through this demo day (and in smaller demos throughout the semester) I likewise want students to see how a practicing cartographer solves problems. While it reinforces some of the skills students have already been taught (but may not have absorbed), it more importantly demonstrates how I, as a professional, make use of the same ideas and technical skills that they have learned. I call this live demo "Bob Ross Day," after the host of The Joy of Painting, who taught many people how to paint by simply letting them watch him do it.

\section{PRACTICAL TUTORIAL ASSIGNMENT}

As a freelancer, I benefit greatly from the fact that the cartographic community is built upon a culture of sharing knowledge. Mappers are always figuring out new tools and techniques, and then using social media, articles, or conference presentations to teach colleagues about them. I want my students to participate in this spirit of sharing by teaching something useful to their lab colleagues. Late in the semester, I require them to turn in a simple one to two page practical cartographic tutorial. The assignment is pretty open: they need to produce a document that will teach their fellow students something that is useful for mapmaking, and that we haven't talked about in class. It could be a data source, a tool in Adobe Illustrator, a trick in ArcMap, etc., as long as it's novel (to them) and practical.

When I first introduced this assignment, I was a little concerned that students wouldn't know what to do. After all, if I was asking them to learn something new, but not saying exactly what that was, might not some of them get lost? I have, however, been pleased to find that almost every student manages to produce something useful and interesting, with no guidance. They have been very good at exploring the wider world of cartography and bringing back useful knowledge. Their tutorials are later shared on the course website, so that everyone can benefit. It's especially encouraging to have a student ask a question in class and then have another student respond, "I wrote a guide about that recently; have a look and let me know if you have any questions!” I draw satisfaction from seeing them 
engaged in the kind of culture of mutual assistance that I benefit from in my own professional work.

\section{ONLINE DISCUSSION}

Whenever I get stuck on a project, or have a technical question, I ask colleagues for help. Wanting to replicate that experience for my students, I set up an online discussion forum using Slack, which is a group chat application popular with a number of cartographers and GIS professionals. While there are public Slack chats on the wider internet, the one I established was accessible only by members of the course. I then encouraged students to post their questions and solicit feedback on their work, so that other students could log in and offer help and advice.

Results with the Slack chat have been mixed. The first semester I tried it, students were fairly engaged with each other, responding to each other's queries a few times per week. Not a great deal of traffic, but the site was definitely seeing regular use throughout the semester. It was very satisfying to see students helping each other out, and becoming teachers of the material they had learned. I and my teaching assistants also chimed in from time to time, to help out on questions that weren't being answered, or suggest alternatives to students' answers.
Unfortunately, the following semester, the Slack chat lay mostly empty. Almost no questions were asked, and any engagement was mostly between myself and the students, rather than peer-to-peer. There were 38 students in that course, as compared with 59 in the prior semester, so perhaps there is some minimum number that's necessary to achieve critical mass. Whatever the cause may be for the decline in use, I think the idea still has merit; however, it may require further experimentation to find a successful formula, and perhaps it is not as sustainable in smaller classes.

\section{A WORK IN PROGRESS}

I hope that these examples will be of some value to you, and perhaps inspire you to iterate upon them. I think these elements combine to produce a course that teaches not only the basics of cartography, but also some of the basics of being a cartographer. Just as with my freelance work, the course is a work in progress, going through multiple drafts: each semester I make small changes, trying new things and adjusting or discarding old ones. In the end, my goal is to ensure that students leave my course ready to be practicing cartographers. I think I'm moving in that direction. 